\title{
Comparison of dual antiplatelet therapy versus oral anticoagulation following transcatheter aortic valve replacement: A retrospective single-center registry analysis
}

\author{
Erik W. Holy, Julia Kebernik, Abdelhakim Allali, \\ Mohamed El-Mawardy, Gert Richardt, Mohamed Abdel-Wahab \\ Cardiology Department, Heart Center, Segeberger Kliniken (Academic Teaching \\ Hospital of the Universities of Kiel, Lübeck and Hamburg), Bad Segeberg, Germany
}

\begin{abstract}
Background: The choice of optimal antithrombotic regimen after transcatheter aortic valve replacement (TAVR) remains a matter of debate. The objective of this study was to compare both efficacy and safety outcomes based on the type of antithrombotic therapy prescribed after TAVR

Methods: This is a retrospective analysis of 514 consecutive patients treated with either dual antiplatelet therapy (DAPT) $(n=315 ; 61.3 \%)$ or oral anticoagulation (OAC) plus clopidogrel ( $n=199 ; 38.7 \%)$ for a minimum of 3 months after TAVR followed by antiplatelet monotherapy or OAC only, respectively. Patients had pre-defined clinical and echocardiographic follow-ups at 30 days, 6 and 12 months. The key efficacy endpoint was a composite of all-cause death, myocardial infarction, stroke and valve thrombosis at 1 year. The key safety endpoint was the occurrence of life-threatening/major bleeding at 1 year. Results: Baseline characteristics did not differ between both groups, except for a higher incidence of atrial fibrillation in the OAC group. No significant differences in both efficacy and safety endpoints were observed at 30 days and 6 months. At 1 year, the key efficacy endpoint occurred in 21.5\% of the DAPT group compared to $19.7 \%$ of the OAC group $(p=0.61)$. The key safety endpoint occurred in $25.1 \%$ and $27.8 \%$, respectively $(p=0.53)$. However, after 1 year valve thrombosis was reported in $8(2.5 \%)$ patients in the DAPT group but not in the OAC group $(p=0.02)$.

Conclusions: OAC after TAVR seems to reduce the risk of clinical valve thrombosis without a statistically significant increase in bleeding complications. (Cardiol J 2017; 24, 6: 649-659)

Key words: oral anticoagulation, platelet aggregation inhibitors, transcatheter aortic valve replacement, valve thrombosis
\end{abstract}

\section{Introduction}

The use of antithrombotic medication following transcatheter aortic valve replacement (TAVR) is currently based on empirical data and mainly derived from clinical experience with coronary stenting and established surgical valve procedures. The risk of thromboembolic events after TAVR have been reported to be the highest within the first days after intervention, in line with the experience gained over the last decades from surgical bioprosthetic valves [1-3]. Current multisociety guidelines recommend a dual antiplatelet regimen defined as a combination of low-dose aspirin and the thienopyridine clopidogrel for at least 3 to 6 months after TAVR [4]. However, data supporting this concept are sparse. A recently published propensity matched analysis of pooled

Address for correspondence: Erik W. Holy, MD, Cardiology Department, Heart Center Segeberger Kliniken GmbH, Am Kurpark 1, 23795 Bad Segeberg, Germany, e-mail: erik.w.holy@gmail.com 


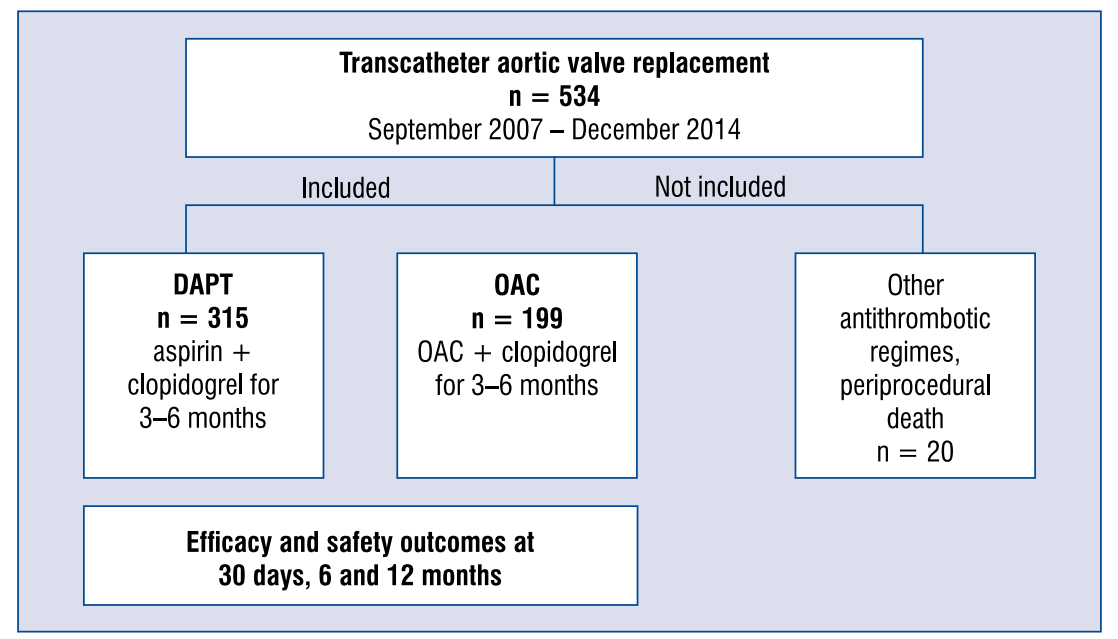

Figure 1. Study design and patient enrolment; DAPT — dual antiplatelet therapy; OAC — oral anticoagulation.

data from 672 TAVR patients treated with either dual antiplatelet therapy (DAPT) or aspirin alone suggests that DAPT is associated with higher rates of bleeding complications compared to aspirin monotherapy, while net adverse ischemic and thrombotic events, including stroke, did not differ between the groups at 30 days [5]. Patients undergoing TAVR with underlying atrial fibrillation (AF) or previous thromboembolic events constitute a particularly challenging population with regard to the post-interventional antithrombotic management. AF has been reported in more than $30 \%$ of the patients considered for TAVR and is associated with a markedly increased risk of mortality and thromboembolic events after valve replacement $[6,7]$. In addition, new onset AF was observed in one third of the patients undergoing continuous electrocardiographic (ECG)-monitoring after TAVR and associated with higher rates of postprocedural embolic events [8]. Identifying the appropriate anticoagulation strategy after TAVR remains an important clinical challenge that has resulted in a large variability of antithrombotic therapies, ranging from triple therapy (DAPT + oral anticoagulation $[\mathrm{OAC}]$ ) to vitamin-K antagonists (VKA) alone [9]. Finally, recent reports on low but clinically relevant incidence of valve thrombosis hindering prosthesis function may further lead to an upheaval in the landscape of thrombotic therapy after TAVR [10-12].

The objective of the present retrospective single center registry analysis was to compare the safety and efficacy outcomes for up to 1 year of either an antiplatelet therapy or OAC after TAVR.

\section{Methods}

\section{Study design}

This retrospective analysis was performed in a single-center prospective cohort study aiming for consecutive enrolment of all patients undergoing TAVR procedures at the Heart Center Segeberger Kliniken, Bad Segeberg, Germany. Data collection was approved by the local ethics committee and informed written consent was obtained from all patients. The current analysis includes 514 consecutive patients who underwent TAVR between September 2007 and December 2014 at the Heart Center Segeberger Kliniken, Germany. Twenty patients were excluded from this analysis because of antithrombotic treatments other than DAPT or OAC, or due to procedure related death (Fig. 1).

\section{Procedure}

Transcatheter aortic valve replacement was performed in all patients with Conformite Européenne marked devices. TAVR procedures were conducted via trans-femoral, trans-subclavian, trans-apical or trans-aortic access sites. Device success was defined according to the Valve Academic Research Consortium 2 (VARC-2) consensus document, which is a technical composite end point including successful vascular access, delivery and deployment of the device and successful retrieval of the delivery system; correct position of the device in the proper anatomical location; intended performance of the prosthetic heart valve (aortic valve area [AVA] $>1.2 \mathrm{~cm}^{2}$, mean aortic valve gradient $<20 \mathrm{~mm} \mathrm{Hg}$, or peak velocity $<3 \mathrm{~m} / \mathrm{s}$, 
without moderate or severe prosthetic valve aortic regurgitation as assessed by post-interventional transthoracic echocardiography [TTE]) and having only 1 valve implanted in the proper anatomical location $[13,14]$.

\section{Antithrombotic regimen following TAVR}

Patients were categorized into two groups based on the antithrombotic therapy established after TAVR. The DAPT group consisted of 315 (61.3\%) patients treated with a combination of oral aspirin and clopidogrel for 3 months followed by lifelong aspirin therapy. The OAC group included $199(38.7 \%)$ patients treated with a combination of an OAC + clopidogrel for 3 months followed by $\mathrm{OAC}$ alone (Fig. 1). In patients treated with concomitant percutaneous coronary intervention (PCI) and drug-eluting stent implantation antithrombotic therapy with DAPT or combination of OAC + clopidogrel was pursued for up to 6 months.

\section{Follow-up and study endpoints}

All patients in the registry had a pre-defined clinical and transthoracic echocardiographic (Vivid 7 Ultrasound Machine and a M4S matrix array sector transducer GE Medical Systems, Milwaukee, WI, USA) follow-up at 30 days, 6 months and 1 year. Additional transesophageal echocardiography (TEE) evaluation was performed in cases of worsening symptoms or suspected valve thrombosis, defined as valve dysfunction (mean transvalvular gradient $>20 \mathrm{~mm} \mathrm{Hg}$, reduction of the AVA to $<1.2 \mathrm{~cm}^{2}$ or new onset more than mild transvalvular regurgitation) or newly apparent mobile mass suspicious of thrombus, irrespective of dysfunction, and in the absence of infection. In addition, multidetector computed tomography (MDCT) examination was performed to further confirm the diagnosis of suspected valve thrombosis. CT examination was done with a second generation dual-source CT scanner (Somatom Definition Flash, Siemens Healthcare, Forchheim, Germany). Contrast enhanced ECG-gated acquisition of the aortic root was performed after injection of iodinated contrast agent with the region of interest placed in the ascending aorta and axial sections of $0.6 \mathrm{~mm}$. All data were transferred to a dedicated post-processing workstation (Syngo Multimodality Workplace, Siemens Healthcare, Forchheim, Germany) for analysis. Images were evaluated for hypoattenuating areas with or without reduced mobility of one or more leaflets identifiable in two different projections. Valve thrombosis was classified based on the timing of diagnosis after TAVR as acute (0-10 days); subacute (11-30 days) or late ( $>1$ month). Pre-specified efficacy endpoints of this study were defined as all-cause death, myocardial infarction (MI), stroke and clinical valve thrombosis at 30 days, 6 months and 1 year. The key efficacy endpoint was a composite of all at 1 year. The key safety endpoint was the occurrence of life threatening or major bleeding at 1 year. Individual endpoints were defined according to the VARC-2 criteria for event definition [14].

\section{Statistical analysis}

Continuous data are reported as mean \pm standard deviation (SD) for normally distributed variables and as median with interquartile range (IQR) for non-normally distributed variables. Categorical variables are reported as number of patients (\% of patients). Normally and nonnormally distributed variables were compared using the Student's t-test or the Mann-Whitney $\mathrm{U}$ test accordingly. Comparisons between categorical variables were performed using the $\chi^{2}$ or Fisher's exact test, wherever appropriate. In order to identify whether the anti-thrombotic treatment strategy was predictive of all-cause mortality, cardiovascular mortality, stroke, major bleeding, or combined efficacy and safety endpoints multivariable binary logistic regression analysis was performed. Variables which reached a $\mathrm{p}$-value $<0.1$ on univariate binary regression analysis were included in the model. Variables are adjusted for age, sex, body mass index (BMI), $\mathrm{AF}$ and staged PCI. All tests were two-tailed and a p-value of $<0.05$ was considered as statistically significant. All the analyses were retrospective and performed using STATA version 14 (STATA Corp., TX, USA) or SPSS (SPSS, Release 22; SPSS Inc, Chicago, IL).

\section{Results}

A total of 514 consecutive patients that underwent TAVR at the indicated institution were included in this study. The mean age of the study population was 80.4 years, $43.7 \%$ were males, with a median logistic EuroScore of $18.49 \%$. In both groups patients had severe aortic stenosis. Echocardiographic characteristics did not differ significantly in either group. The mean ( \pm SD) gradient was $46 \pm 16.8 \mathrm{~mm} \mathrm{Hg}$ in the DAPT group and $44 \pm 16.8 \mathrm{~mm} \mathrm{Hg}$ in the OAC group $(\mathrm{p}=0.27)$. The indexed AVA was $0.41 \pm 0.2$ $\mathrm{cm}^{2} / \mathrm{m}^{2}$ and $0.39 \pm 0.2 \mathrm{~cm}^{2} / \mathrm{m}^{2}(\mathrm{p}=0.28)$. The two groups had similar baseline characteristics 
Table 1. Baseline characteristics of the study population.

\begin{tabular}{lccc}
\hline & DAPT $(\mathbf{n}=315)$ & OAC (n = 199) & P \\
\hline Age [years], mean \pm SD & $80.4 \pm 7.0$ & $80.6 \pm 5.7$ & 0.73 \\
Female & $181(57.6 \%)$ & $107(54 \%)$ & 0.43 \\
Body mass index, mean \pm SD & $26.4 \pm 4.9$ & $27.5 \pm 4.8$ & $<\mathbf{0 . 0 1}$ \\
Diabetes mellitus & $81(25.8 \%)$ & $59(29.8 \%)$ & 0.32 \\
Hypertension & $279(88.8 \%)$ & $172(86.9 \%)$ & 0.49 \\
Hyperlipidemia & $179(57 \%)$ & $116(58.6 \%)$ & 0.72 \\
Atrial fibrillation & $33(10.5 \%)$ & $137(69.2 \%)$ & $<\mathbf{0 . 0 1}$ \\
Previous stroke & $35(11.1 \%)$ & $24(12.1 \%)$ & 0.23 \\
CHA ${ }_{2}$ DS ${ }_{2}$-VASc-score, mean \pm SD & $4.8 \pm 1.33$ & $4.6 \pm 1.33$ & 0.47 \\
Coronary artery disease & $215(68.5 \%)$ & $126(63.6 \%)$ & 0.25 \\
Staged PCl & $126(40.1 \%)$ & $56(28.3 \%)$ & $<\mathbf{0 . 0 1}$ \\
Previous CABG & $64(20.4 \%)$ & $31(15.6 \%)$ & 0.18 \\
Carotid artery disease & $44(14 \%)$ & $22(11.1 \%)$ & 0.34 \\
Peripheral vascular disease & $46(14.6 \%)$ & $32(16.2 \%)$ & 0.64 \\
EuroScore Logistic, median (IOR) & $18.5(11.4-29.9)$ & $18.2(12.5-31.0)$ & 0.92
\end{tabular}

DAPT — dual antiplatelet therapy; OAC — oral anticoagulation; SD — standard deviation, $\mathrm{PCl}$ - percutaneous coronary intervention; CABG — coronary artery bypass graft; IQR — interquartile range

Table 2. Procedural characteristics.

\begin{tabular}{lccc}
\hline & DAPT $(\mathbf{n}=315)$ & OAC (n = 199) & P \\
\hline Access route: & & & 1.0 \\
Transfemoral & $308(97.8 \%)$ & $3(1.5 \%)$ & 0.38 \\
Transapical & $2(0.6 \%)$ & $0(0.0 \%)$ & 0.28 \\
Subclavian & $3(0.9 \%)$ & $1(0.5 \%)$ & 1.0 \\
Transaortic & $2(0.6 \%)$ & $104(52.3 \%)$ & 0.20 \\
Self-expandable valve prosthesis & $183(58.1 \%)$ & $79(39.7 \%)$ & 0.51 \\
Balloon-expandable valve prosthesis, & $115(36.5 \%)$ & $16(8.0 \%)$ & 0.27 \\
Lotus valve prosthesis & $17(5.4 \%)$ & $174(87.4 \%)$ & 0.51 \\
Device success & $268(85.1 \%)$ & $11(5.5 \%)$ & 0.67 \\
Valve-in-valve procedure & $14(4.4 \%)$ & & 0.83 \\
Periinterventional vascular complications: & & $9(4.5 \%)$ & 0.12 \\
Minor & $16(5.1 \%)$ & $16(8.1 \%)$ & \\
Major & $14(4.4 \%)$ & & \\
\hline
\end{tabular}

DAPT — dual antiplatelet therapy; OAC — oral anticoagulation

(Table 1), except for a higher incidence of AF in the OAC group $(10.5 \%$ vs. $69.2 \%$; $<<0.01)$ and a higher rate of staged $\mathrm{PCI}$ in patients undergoing TAVR in the DAPT group ( $40.1 \%$ vs. $28.3 \%$; $\mathrm{p}=0.01)$. Procedural characteristics did not differ between either group (Table 2). At discharge, $315(61.3 \%)$ patients received DAPT and 199 $(38.7 \%)$ a combination of OAC and clopidogrel
( $\mathrm{n}=188$ phenoprocoumon, $\mathrm{n}=7$ rivaroxaban, $\mathrm{n}=4$ dabigatran) following TAVR (Fig. 1).

Outcomes at 30 days

At 30 days, all-cause mortality occurred in a total of $18(3.5 \%)$ patients, $11(3.5 \%)$ in the DAPT group and $7(3.5 \%)$ in the OAC group $(\mathrm{p}=0.98)$ (Table 3). Cardiovascular mortality was reported in 
Table 3. Outcomes at 30 days.

\begin{tabular}{lccc}
\hline & DAPT $(\mathbf{n}=315)$ & OAC (n = 199) & P \\
\hline All cause death & $11(3.5 \%)$ & $7(3.5 \%)$ & 0.98 \\
Cardiovascular death & $11(3.5 \%)$ & $5(2.5 \%)$ & 0.61 \\
Myocardial infarction & $3(0.9 \%)$ & $1(0.5 \%)$ & 1.0 \\
Stroke, all & $12(3.8 \%)$ & $7(3.5 \%)$ & 0.86 \\
Transcatheter heart valve thrombosis & $1(0.3 \%)$ & $0(0 \%)$ & 1.0 \\
Combined efficacy endpoint & $26(8.2 \%)$ & $13(6.5 \%)$ & 0.47 \\
Life threatening bleeding & $23(7.3 \%)$ & $19(9.6 \%)$ & 0.36 \\
Major bleeding & $53(16.8 \%)$ & $30(15.1 \%)$ & 0.60 \\
Combined safety endpoint & $76(24.1 \%)$ & $49(24.6 \%)$ & 0.88 \\
\hline
\end{tabular}

DAPT — dual antiplatelet therapy; OAC — oral anticoagulation

Table 4. Outcomes at 6 months.

\begin{tabular}{lccc}
\hline & DAPT $(\mathbf{n}=315)$ & OAC (n = 199) & P \\
\hline All cause death & $25(7.9 \%)$ & $24(12.0 \%)$ & 0.15 \\
Cardiovascular death & $16(5.0 \%)$ & $14(7.0 \%)$ & 0.12 \\
Myocardial infarction & $5(1.6 \%)$ & $1(0.5 \%)$ & 0.41 \\
Stroke, all & $14(4.4 \%)$ & $8(4.0 \%)$ & 0.83 \\
Transcatheter heart valve thrombosis & $6(1.9 \%)$ & $0(0 \%)$ & 0.08 \\
Combined efficacy endpoint & $48(15.2 \%)$ & $29(14,6 \%)$ & 0.86 \\
Major bleeding & $55(17.5 \%)$ & $33(16.5 \%)$ & 0.80 \\
Combined safety endpoint & $76(24.1 \%)$ & $51(25.6 \%)$ & 0.64 \\
\hline
\end{tabular}

DAPT — dual antiplatelet therapy; OAC — oral anticoagulation

$3.5 \%$ of the patients in the DAPT group and $2.5 \%$ in the OAC group $(\mathrm{p}=0.61)$. There was no significant difference between either group in the incidence of $\mathrm{MI}(0.9 \%$ vs. $0.5 \%, \mathrm{p}=1.0)$, stroke $(3.8 \%$ vs. $3.5 \%, \mathrm{p}=0.86)$ or valve thrombosis $(0.3 \%$ vs. $0 \%$, $\mathrm{p}=1.0$ ) at 30 -days. Also safety endpoints did not differ between either group: life-threatening bleeding $(7.3 \%$ vs. $9.5 \%, \mathrm{p}=0.36)$ and major bleeding $(16.8 \%$ vs. $15.1 \%, \mathrm{p}=0.60)$.

\section{Outcomes at 6 months}

At 6 months, all-cause mortality was reported in $25(7.9 \%)$ patients in the DAPT group and 24 $(12.1 \%)$ patients in the OAC group $(\mathrm{p}=0.15)$ (Table 4). There was no significant difference between DAPT and OAC groups in the incidence of MI $(1.6 \%$ vs. $0.5 \%, \mathrm{p}=0.41)$ and stroke $(4.5 \%$ vs. $4.1 \%, \mathrm{p}=0.83)$. Although valve thrombosis was only reported in the DAPT group, there was no statistically significant difference $(1.9 \%$ vs. $0 \%, \mathrm{p}=0.08$ ) between the groups after 6 months. Combined efficacy $(15.3 \%$ vs. $14.6 \%, \mathrm{p}=0.8)$ and safety ( $24.4 \%$ vs. $26.1 \%, \mathrm{p}=0.64)$ endpoints at 6 months did not differ significantly (Table 4 ).

\section{Safety and efficacy endpoints at 1 year}

At 1 year all-cause mortality had occurred in a total of 74 (14.4\%) patients. 39 (12.4\%) deaths were reported in the DAPT group and $35(17.6 \%)$ in the OAC group (Fig. 2). Even though a trend towards a higher mortality rate could be observed in the OAC group, this increase did not reach statistical significance $(p=0.09)$. Furthermore, there was no difference in $\mathrm{MI}(2.9 \%$ vs. $0.5 \%, \mathrm{p}=0.09)$ and stroke $(5.2 \%$ vs. $4.1 \%, \mathrm{p}=0.67)$ rates between the DAPT and OAC groups. After 1 year, 8 (2.5\%) cases of valve thrombosis were documented in the group of patients on antiplatelet treatment, while in patients on $\mathrm{OAC}$ no valve thrombosis was reported ( $2.5 \%$ vs. $0 \%, \mathrm{p}=0.02)$. Combined key efficacy $(21.5 \%$ vs. $19.7 \%, \mathrm{p}=0.61)$ and safety $(25.1 \%$ vs. $27.8 \%, \mathrm{p}=0.53$ ) endpoints at 1 year did not differ between the groups (Fig. 3). An adjusted logistic regression analysis confirmed that despite a trend 


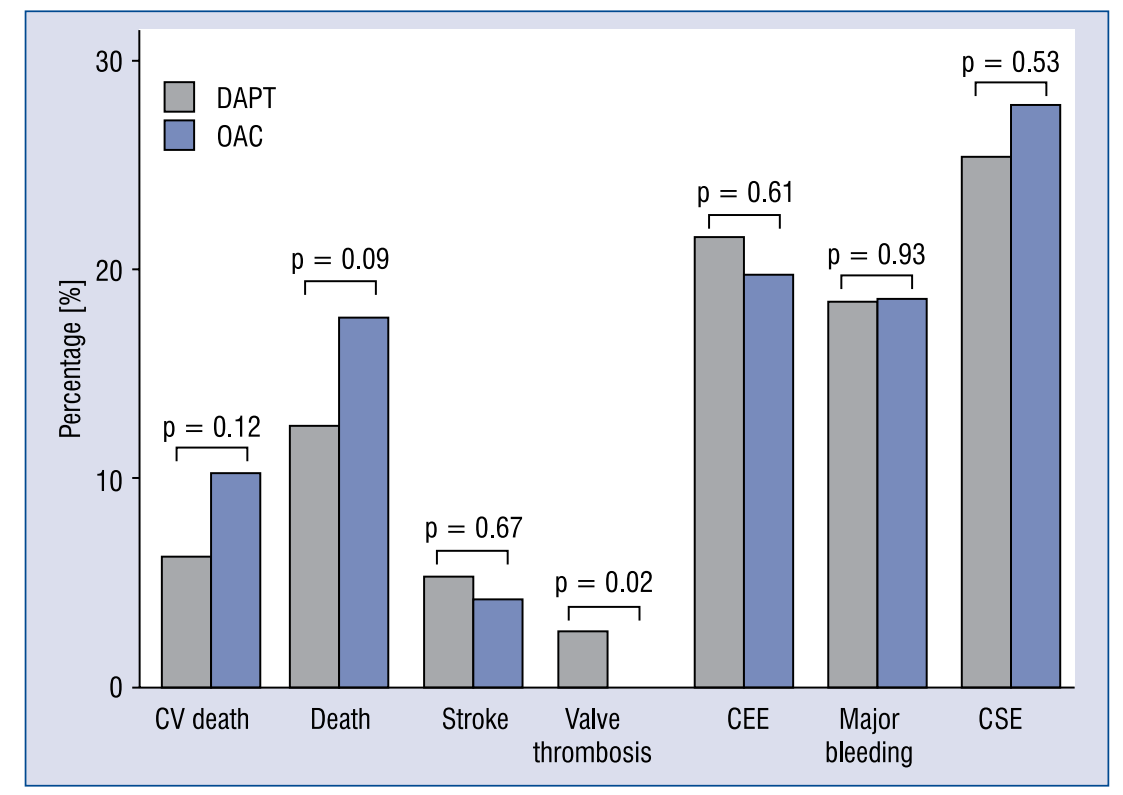

Figure 2. Summary of defined endpoints and outcomes at 1 year in the dual antiplatelet therapy (DAPT) and oral anticoagulation (OAC) group; CEE — combined efficacy endpoint; CSE — combined safety endpoint; CV — cardiovascular.

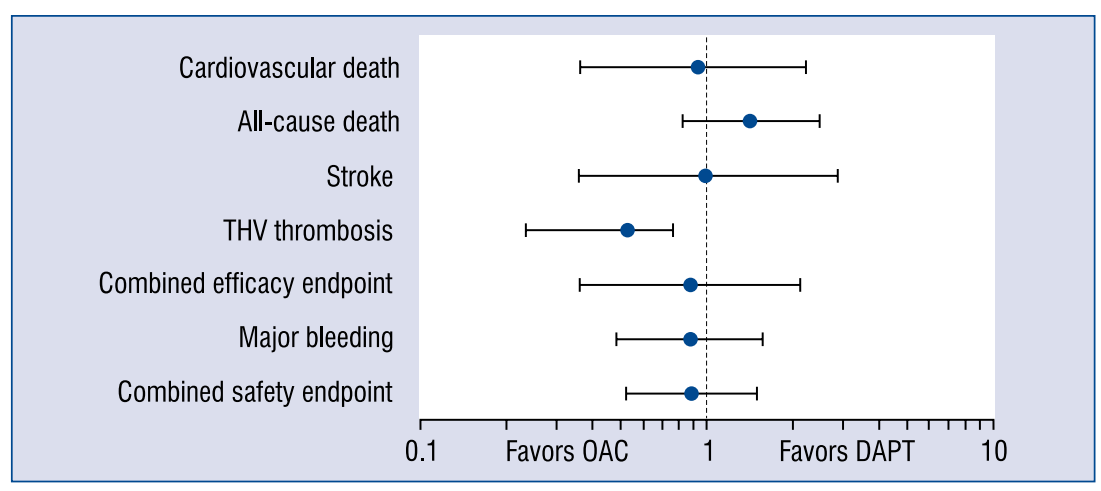

Figure 3. Adjusted interaction between dual antiplatelet therapy (DAPT) or oral anticoagulation (OAC) after transcatheter aortic valve replacement on outcomes at one year. Variables are adjusted for age, sex, body mass index, atrial fibrillation and staged percutaneous coronary intervention. Odds ratios are displayed with $95 \%$ confidence interval on a logarithmic scale; THV — transcatheter heart valve.

towards an increase in all-cause mortality after 1 year observed in patients under OAC there was no overall statistically significant difference in the occurrence rates of the predefined endpoints (Fig. 3). However, OAC reduced the risk of valve thrombosis independent of age, sex, BMI, AF and whether staged PCI was performed (OR 0.53; 95\% CI 0.23-0.76).

\section{Clinical features of transcatheter heart valve thrombosis}

In the current analysis transcatheter heart valve (THV) thrombosis was reported in 8 patients with a mean age of 78.7 years. Median and mean time to diagnosis of THV thrombosis were 181 (IQR 176-263) days and 196 days, respectively. THV thrombosis was considered to be subacute in 1 and late in 7 patients (Table 5). All 8 cases of THV thrombosis occurred in the DAPT group. While 2 patients were still under DAPT (aspirin/ /clopidogrel) 6 patients were under aspirin monotherapy at the point in time of diagnosis of THV thrombosis. Cases of valve thrombosis included 7 patients who had undergone TAVR with a balloon expandable THV and 1 patient treated with a selfexpandable THV. Three cases of THV thrombosis 


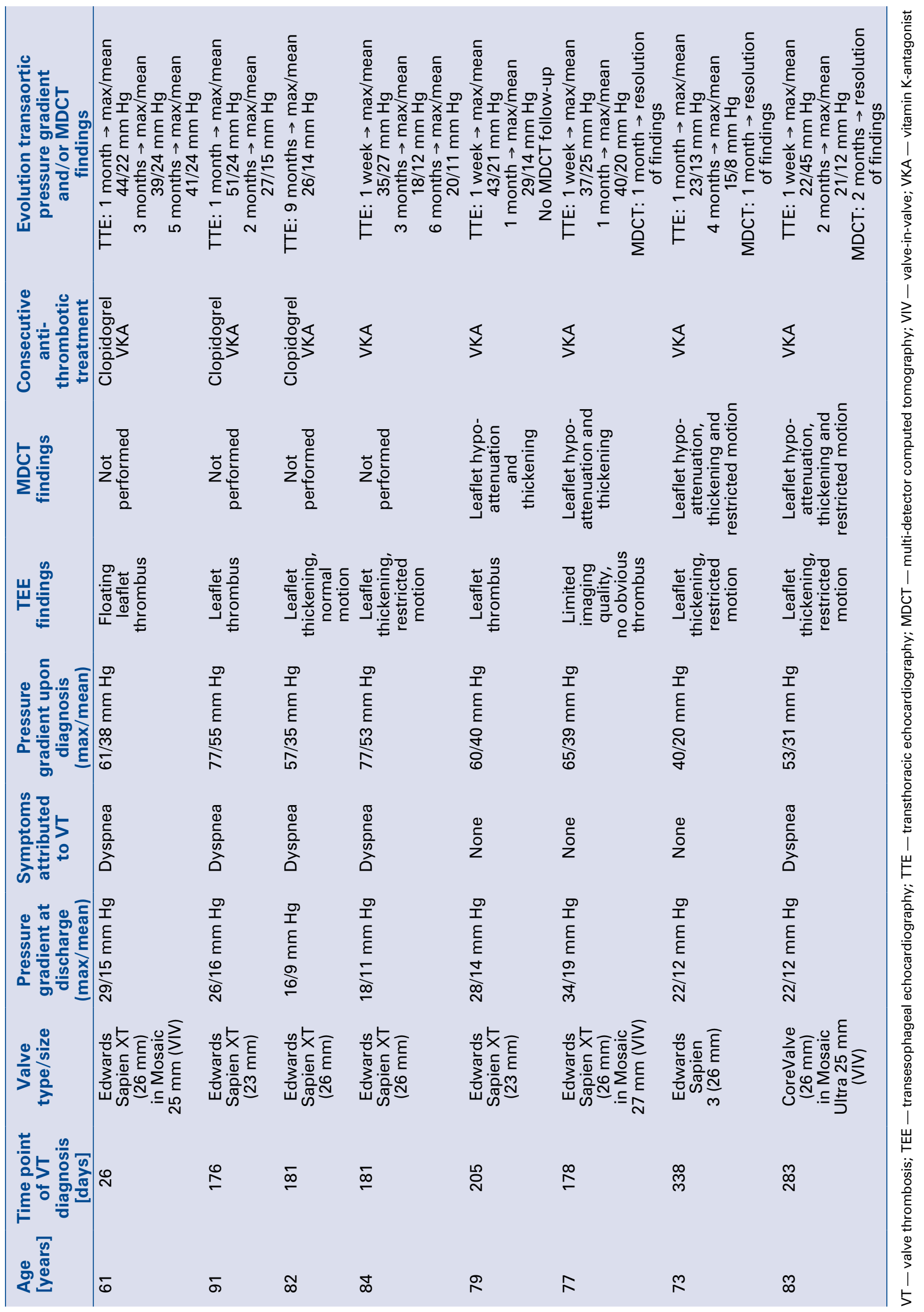


occurred in patients who underwent TAVR for treatment of degenerated surgical bioprosthesis (valve-in-valve). Worsening dyspnea was the leading clinical manifestation of THV thrombosis and was observed in 5 patients. In the other 3 patients no clinical symptom could be attributed to the occurrence of THV thrombosis. No case of stroke, MI or peripheral embolism could be associated to THV thrombosis. Elevated transvalvular gradients was the leading finding in all patients on TTE examination. The peak and mean transaortic gradients at the time of diagnosis of THV thrombosis were $61 \mathrm{~mm} \mathrm{Hg}$ and $39 \mathrm{~mm} \mathrm{Hg}$, respectively. TEE was performed in all patients with suspected THV thrombosis. TEE findings included restricted leaflet mobility $(\mathrm{n}=3)$, thrombotic mass $(\mathrm{n}=3)$ and thickened leaflets $(n=4)$ (Table 5$)$. In 1 case TEE was inconclusive. CT examination was performed in 4 patients and demonstrated hypoattenuated lesions of the leaflets and restricted leaflet motion consistent with THV thrombosis. All patients diagnosed with THV thrombosis were treated with VKA in therapeutic doses. Three patients were treated in addition with clopidogrel. Under OAC follow-up TTE examinations documented a decrease of the transvalvular gradients in all patients. Follow up CT was performed in 3 patients $1(n=2)$ and $2(n=1)$ months after initiation of anticoagulant therapy confirmed resolution of the hypoattenuated lesions and leaflet thickening with restoration of leaflet motion. After resolution of THV thrombosis OAC was ceased in 3 patients while VKA treatment was continued in 5 patients. No recurrence of THV thrombosis was reported.

\section{Modification of therapeutic regimen}

During the observational period of 1 year, 37 patients underwent modification of their therapeutic regimen. 19 patients of the DAPT group switched to OAC and 18 patients of the OAC group switched to DAPT due to clinical indications. Follow-up classification and statistical analysis was based on an intention-to-treat analysis.

\section{Discussion}

This retrospective analysis of a single center registry compared outcomes of two antithrombotic regimen following TAVR. After a follow-up period of 1 year no significant differences in predefined cumulative efficacy and safety endpoints were observed between an antithrombotic strategy based on early phase DAPT for 3-6 months according to current guideline recommendations and OAC.
However, clinical valve thrombosis after TAVR occurred only in the DAPT group and was successfully treated by the establishment of OAC.

Most cardioembolic events after TAVR were reported to occur within the first days after TAVR [15]. In the present study, the incidence of all-cause mortality, stroke and MI at 30 days was comparable to results previously published in prospective landmark trials as well as nationwide registries [16-20]. Post interventional DAPT or OAC resulted in similar rates of MI, stroke and all-cause mortality up to 6 months after TAVR thereby underscoring the efficacy of both therapeutic regimens. After 6 months, patients in the DAPT group were placed on single antiplatelet therapy (aspirin) while anticoagulation was pursued in the OAC group due to comorbidities, in particular paroxysmal or permanent AF. One year after TAVR, aspirin monotherapy also yielded similar results as compared to OAC regarding predefined efficacy endpoints. Hence, the present results are in line with previous studies strongly supporting an interruption of DAPT after a period of 3-6 months in TAVR patients who do not have any further indication for DAPT such as recent acute coronary syndrome ( $<12$ months) or coronary stent implantation ( $<6$ months) [5, 21, $22]$. Interestingly, after 1 year, and despite adjustment for the occurrence of $\mathrm{AF}$ and other variables, a trend towards an increased all-cause mortality in the OAC group could be observed. Considering the limited size of the study population as well as the retrospective nature of the study, this observation requires careful interpretation.

In the relatively young era of percutaneous aortic valve replacement, an increasing number of reports has emerged describing cases of clinically relevant subacute post-TAVR valve thrombosis and recently identified reduced leaflet motion in MDCT, which may correspond to a subclinical surrogate of valve thrombosis $[10,11,23-25]$. These reports about post-TAVR valve thrombosis and hindered leaflet function have generated debate about the clinical relevance of this phenomenon. In the present study, valve thrombosis was diagnosed based on routine echocardiographic follow-up examination in $1.5 \%$ of the total population and as often as $2.5 \%$ in patients initially treated with DAPT. Diagnosis was based on echocardiographic parameters demonstrating a significant increase in transvalvular pressure gradients or thrombotic mass and confirmed by MDCT findings reporting thickening of the leaflets and reduced leaflet motion. THV thrombosis manifested as worsening of dyspnoea in 5 of 8 patients while it was not as- 
sociated to any clinical symptoms in 3 patients. In patients diagnosed with THV thrombosis, DAPT was replaced by OAC for at least 3 months and follow-up intervals were shortened. Follow-up echocardiography as well as clinical examination documented restoration of valve function under OAC by decreases of peak and mean transvalvular gradients over valve prosthesis, resolution of thrombotic mass as well as improvement of clinical symptoms in all patients. Interestingly, in contrast to the DAPT group, no cases of valve thrombosis were diagnosed in any of the patients on $\mathrm{OAC}$ and logistic regression analysis demonstrated that OAC was associated with a significantly lower risk of developing THV thrombosis. This observation is in line with current reports in the literature suggesting that OAC but not antiplatelet therapy prevents valve thrombosis [26-28]. Further studies are required in order to better characterize this subgroup of patients and to identify potential predictors which reveal those patients who may benefit from OAC after TAVR apart from other indications for anticoagulant therapy.

Hemorrhagic complications are frequent, and major or life-threatening bleeding after TAVR occur in $20-30 \%$ and $15 \%$, respectively [16, 17, 29]. Regardless of the underlying mechanisms, disabling or life-threatening bleeding is an important predictor of acute and late mortality in patients undergoing TAVR. Predictors of bleeding complications have been identified by several groups [30, 31]. Among them, vascular or major intraprocedural complications, female sex, and baseline anemia have been most frequently reported. While periprocedural bleeding is driven mainly by anatomical and technical considerations, major and life-threatening bleeding occurring at later points in time appear to be related mainly to patient bleeding susceptibility, triggered by the antithrombotic agent used. In the current analysis the composite safety endpoint occurred mainly within the first 6 months after TAVR in both groups. This observation strongly suggests that both DAPT and the combination of $\mathrm{OAC}$ with a single antiplatelet drug such as clopidogrel results in a significant increase of hemorrhagic complications after TAVR in a patient population that is at particular high risk for bleeding due to comorbidities and frailty. At 1 year the incidence of the composite safety endpoint was only slightly higher in both groups as compared to the 6 months time point and was mostly related to the fact that after a maximum of 6 months thienopyridine therapy was ceased in patients without an indication for further long-term clopidogrel treat- ment such as acute coronary syndrome. Previous studies and registries have reported a short-term hazard related to bleeding complications that were most apparent during the first 3 months of initiation of either VKA plus a single antiplatelet drug or DAPT treatment [32, 33]. A most recent meta-analysis excluding patients on OAC demonstrated that aspirin monotherapy while being as efficient as DAPT in preventing post-interventional thromboembolic complications and was superior to DAPT with regard to bleeding complications [5]. Taken together these observations may challenge the current practice recommending DAPT for 3-6 months after TAVR but these results need to be confirmed by currently ongoing prospective randomized trials. With regard to the management of antithrombotic therapy after TAVR in patients on $\mathrm{OAC}$, further data reporting is warranted. In this retrospective analysis patients on $\mathrm{OAC}$ were treated with clopidogrel for at least 3 months after TAVR according to current recommendations [4]. Since the majority of the bleeding events occurred in this group within the first 6 months, the net benefit of adding a platelet inhibitor on top of $\mathrm{OAC}$ may be questioned. As for DAPT, prospective randomized trials are required to define the most appropriate antithrombotic post-TAVR therapy in patients on OAC. Finally, while the newer direct oral anticoagulants have proven to be safer and at least as efficient as VKA in the prevention of thromboembolic events in patients with underlying non-valvular AF, their role in patients undergoing TAVR remains to be defined.

\section{Limitations of the study}

As for every retrospective cohort analysis limitations such as patient and treatment selection biases need to be considered. The reporting in this study followed the VARC-2 criteria and was predefined. TEE and MDCT examinations were only performed in patients with suspected valve thrombosis defined by worsening transvalvular gradient on follow-up TTE or new onset of symptoms. Hence, it seems likely that the incidence of subclinical valve thrombosis which may be more accurately diagnosed by routine MDCT or TEE examination may have been underestimated in this retrospective study.

\section{Conclusions}

This single center analysis suggests that DAPT and OAC are associated with similar clinical outcomes at 1 year with regard to predefined 
safety and efficacy endpoints in patients undergoing TAVR. In addition, OAC seems to reduce the risk of clinical valve thrombosis without a significant increase in major bleeding complications. Further randomized controlled trials assessing the optimal antithrombotic therapy after TAVR are both justified and needed.

Conflict of interest: Erik W. Holy - EAPCI training grant (Edwards Lifesciences), research grant University of Zurich, Switzerland; Gert Richardt and Mohamed Abdel-Wahab - research grant SJM and Biotronik lecture fees Edwards Lifesciences and Boston Scientific.

\section{References}

1. Möllmann H, Bestehorn K, Bestehorn M, et al. In-hospital outcome of transcatheter vs. surgical aortic valve replacement in patients with aortic valve stenosis: complete dataset of patients treated in 2013 in Germany. Clin Res Cardiol. 2016; 105(6): 553-559, doi: 10.1007/s00392-016-0962-4, indexed in Pubmed: 26830097.

2. Abdel-Wahab M, Jose J, Richardt G. Transfemoral TAVI devices: design overview and clinical outcomes. EuroIntervention. 2015; 11 Suppl W: W114-W118, doi: 10.4244/EIJV11SWA33, indexed in Pubmed: 26384175.

3. Messé SR, Acker MA, Kasner SE, et al. Determining Neurologic Outcomes from Valve Operations (DeNOVO) Investigators. Stroke after aortic valve surgery: results from a prospective cohort. Circulation. 2014; 129(22): 2253-2261, doi: 10.1161/CIRCULATIONAHA.113.005084, indexed in Pubmed: 24690611.

4. Nishimura RA, Otto CM, Bonow RO, et al. ACC/AHA Task Force Members. 2014 AHA/ACC Guideline for the Management of Patients With Valvular Heart Disease: a report of the American College of Cardiology/American Heart Association Task Force on Practice Guidelines. Circulation. 2014; 129(23): e521-e643, doi: 10.1161/CIR.0000000000000031, indexed in Pubmed: 24589853.

5. Hassell ME, Hildick-Smith D, Durand E, et al. Antiplatelet therapy following transcatheter aortic valve implantation. Heart. 2015; 101(14): 1118-1125, doi: 10.1136/heartjnl-2014-307053, indexed in Pubmed: 25948421.

6. Yankelson L, Steinvil A, Gershovitz L, et al. Atrial fibrillation, stroke, and mortality rates after transcatheter aortic valve implantation. Am J Cardiol. 2014; 114(12): 1861-1866, doi: 10.1016/j.amjcard.2014.09.025, indexed in Pubmed: 25438914.

7. Stortecky S, Buellesfeld L, Wenaweser P, et al. Atrial Fibrillation and Aortic Stenosis: Impact on Clinical Outcomes Among Patients Undergoing Transcatheter Aortic Valve Implantation. Circ Cardiovasc Interv. 2013; 6(1): 77-84, doi: 10.1161/circinterventions.112.000124.

8. Amat-Santos IJ, Rodés-Cabau J, Urena M, et al. Incidence, predictive factors, and prognostic value of new-onset atrial fibrillation following transcatheter aortic valve implantation. J Am Coll Cardiol. 2012; 59(2): 178-188, doi: 10.1016/j.jacc.2011.09.061, indexed in Pubmed: 22177537.

9. Nijenhuis VJ, Stella PR, Baan J, et al. Antithrombotic therapy in patients undergoing TAVI: an overview of Dutch hospitals. Neth Heart J. 2014; 22(2): 64-69, doi: 10.1007/s12471-013-0496-6, indexed in Pubmed: 24287807.
10. Latib A, Naganuma T, Abdel-Wahab M, et al. Treatment and clinical outcomes of transcatheter heart valve thrombosis. Circ Cardiovasc Interv. 2015; 8(4), doi: 10.1161/CIRCINTERVENTIONS.114.001779, indexed in Pubmed: 25873727.

11. Leetmaa T, Hansson NC, Leipsic J, et al. Early aortic transcatheter heart valve thrombosis: diagnostic value of contrast-enhanced multidetector computed tomography. Circ Cardiovasc Interv. 2015; 8(4), doi: 10.1161/CIRCINTERVENTIONS.114.001596, indexed in Pubmed: 25873726.

12. Huynh K. Thrombosis: Leaflet motion after TAVI or SAVR. Nat Rev Cardiol. 2015; 12(12): 683, doi: 10.1038/nrcardio.2015.170, indexed in Pubmed: 26503406.

13. Baumgartner H, Hung J, Bermejo J, et al. American Society of Echocardiography, European Association of Echocardiography, EAE/ASE. Echocardiographic assessment of valve stenosis: EAE/ASE recommendations for clinical practice. Eur J Echocardiogr. 2009; 10(1): 1-25, doi: 10.1093/ejechocard/jen303, indexed in Pubmed: 19065003.

14. Kappetein A, Head S, Généreux P, et al. Updated standardized endpoint definitions for transcatheter aortic valve implantation: the Valve Academic Research Consortium-2 consensus document†. Eur Heart J. 2012; 33(19): 2403-2418, doi: 10.1093/eurhearti/ehs255.

15. Tay ELW, Gurvitch R, Wijesinghe N, et al. A high-risk period for cerebrovascular events exists after transcatheter aortic valve implantation. JACC Cardiovasc Interv. 2011; 4(12): 1290-1297, doi: 10.1016/j.jcin.2011.08.012, indexed in Pubmed: 22192370 .

16. Smith C, Leon M, Mack M, et al. Transcatheter versus Surgical Aortic-Valve Replacement in High-Risk Patients. N Engl J Med. 2011; 364(23): 2187-2198, doi: 10.1056/nejmoa1103510.

17. Reynolds MR, Lei Y, Wang K, et al. CoreValve US High Risk Pivotal Trial Investigators, U.S. CoreValve Clinical Investigators. Transcatheter aortic-valve replacement with a self-expanding prosthesis. N Engl J Med. 2014; 370(19): 1790-1798, doi: 10.1056/NEJMoa1400590, indexed in Pubmed: 24678937.

18. Hamm CW, Möllmann H, Holzhey D, et al. GARY-Executive Board. The German Aortic Valve Registry (GARY): in-hospital outcome. Eur Heart J. 2014; 35(24): 1588-1598, doi: 10.1093/ eurheartj/eht381, indexed in Pubmed: 24022003.

19. Abdel-Wahab M, Zahn R, Horack M, et al. Transcatheter aortic valve implantation in patients with and without concomitant coronary artery disease: comparison of characteristics and early outcome in the German multicenter TAVI registry. Clin Res Cardiol. 2012; 101(12): 973-981, doi: 10.1007/s00392-012-0486-5, indexed in Pubmed: 22772776.

20. Ludman PF, Moat N, Belr MA, et al. Belder MA de, Transcatheter aortic valve implantation in the United Kingdom: temporal trends, predictors of outcome, and 6-year follow-up: a report from the UK Transcatheter Aortic Valve Implantation (TAVI) Registry, 2007 to 2012. Circulation. 2015; 131: 1181-1190.

21. Magkoutis NA, Fradi S, Azmoun A, et al. Antiplatelet Therapy in TAVI: Current Clinical Practice and Recommendations. Curr Pharm Des. 2016; 22(13): 1888-1895, indexed in Pubmed: 26898915.

22. Gandhi S, Schwalm JDR, Velianou JL, et al. Comparison of Dualantiplatelet Therapy to Mono-antiplatelet Therapy After Transcatheter Aortic Valve Implantation: Systematic Review and Meta-analysis. Can J Cardiol. 2015; 31(6): 775-784, doi: 10.1016/ j.cjca.2015.01.014, indexed in Pubmed: 25913473. 
23. Neylon A, Degrell P, Troussier X, et al. Very Early Thrombosis of Sapien 3 Valve. JACC Cardiovasc Interv. 2016; 9(4): e35-e36, doi: 10.1016/j.jcin.2015.11.004, indexed in Pubmed: 26777330.

24. De Marchena E, Mesa J, Pomenti S, et al. Thrombus formation following transcatheter aortic valve replacement. JACC Cardiovasc Interv. 2015; 8(5): 728-739, doi: 10.1016/j.jcin.2015.03.005, indexed in Pubmed: 25946447.

25. Makkar RR, Fontana G, Søndergaard L, et al. Possible Subclinical Leaflet Thrombosis in Bioprosthetic Aortic Valves. N Engl J Med. 2015; 373(21): 2015-2024, doi: 10.1056/NEJMoa1509233, indexed in Pubmed: 26436963.

26. Latib A, Messika-Zeitoun D, Maisano F, et al. Reversible Edwards Sapien XT dysfunction due to prosthesis thrombosis presenting as early structural deterioration. J Am Coll Cardiol. 2013; 61(7): 787789, doi: 10.1016/j.jacc.2012.10.016, indexed in Pubmed: 23219300.

27. Cota L, Stabile E, Agrusta M, et al. Bioprostheses "Thrombosis” After Transcatheter Aortic Valve Replacement. J Am Coll Cardiol. 2013; 61(7): 789-791, doi: 10.1016/j.jacc.2012.11.042.

28. Descoux J, Gautier-Pignonblanc P, Innorta A, et al. Effectiveness of anticoagulant therapy in the treatment of post-TAVI bioprosthetic thrombosis. J Cardiothorac Surg. 2015; 10: 50, doi: 10.1186/s13019-015-0254-5, indexed in Pubmed: 25886753.

29. Kochman J, Rymuza B, Huczek Z, et al. Incidence, Predictors and Impact of Severe Periprocedural Bleeding According to VARC-2 Criteria on 1-Year Clinical Outcomes in Patients After Transcath- eter Aortic Valve Implantation. Int Heart J. 2016; 57(1): 35-40, doi: 10.1536/ihj.15-195, indexed in Pubmed: 26673439.

30. Konigstein M, Havakuk O, Arbel Y, et al. Impact of Hemoglobin Drop, Bleeding Events, and Red Blood Cell Transfusions on Long-term Mortality in Patients Undergoing Transaortic Valve Implantation. Can J Cardiol. 2015: s0828-282X: 01577-9.

31. Stortecky S, Stefanini GG, Pilgrim T, et al. Validation of the Valve Academic Research Consortium Bleeding Definition in Patients With Severe Aortic Stenosis Undergoing Transcatheter Aortic Valve Implantation. J Am Heart Assoc. 2015; 4: e002135.

32. Lamberts M, Olesen JB, Ruwald MH, et al. Bleeding after initiation of multiple antithrombotic drugs, including triple therapy, in atrial fibrillation patients following myocardial infarction and coronary intervention: a nationwide cohort study. Circulation. 2012; 126(10): 1185-1193, doi: 10.1161/CIRCULATIONAHA.112.114967, indexed in Pubmed: 22869839.

33. Connolly S, Pogue J, Hart R, et al. ACTIVE Writing Group of the ACTIVE Investigators. Clopidogrel plus aspirin versus oral anticoagulation for atrial fibrillation in the Atrial fibrillation Clopidogrel Trial with Irbesartan for prevention of Vascular Events (ACTIVE W): a randomised controlled trial. Lancet. 2006; 367(9526): 1903-1912, doi: 10.1016/S0140-6736(06)68845-4, indexed in Pubmed: 16765759. 